\title{
Cancerous Inhibitor of PP2A Silencing Inhibits Proliferation and Promotes Apoptosis in Human Multiple Myeloma Cells
}

\author{
Xi Yang, Yaping Zhang, Hong Liu, and Zenghua Lin \\ Department of Hematology, Affiliated Hospital of Nantong University, Nantong 226001, China \\ Correspondence should be addressed to Hong Liu; hongliu63@126.com and Zenghua Lin; linzenghua_xy@163.com
}

Received 19 January 2016; Revised 13 March 2016; Accepted 20 March 2016

Academic Editor: Stefan Knapp

Copyright (C) 2016 Xi Yang et al. This is an open access article distributed under the Creative Commons Attribution License, which permits unrestricted use, distribution, and reproduction in any medium, provided the original work is properly cited.

\begin{abstract}
Multiple myeloma is the second most prevalent type of blood cancer, representing approximately $1 \%$ of all cancers and $2 \%$ of all cancer deaths. There is therefore a strong need to identify critical targets in multiple myeloma neoplasia and progression. Cancerous inhibitor of PP2A (CIP2A) is a human oncoprotein that regulates cancer cell viability and anchorage-independent growth and induces apoptosis. The present study investigated CIP2A function in the human multiple myeloma cell lines RPMI8226 and NCI-H929 to determine whether it can serve as a potential therapeutic target. CIP2A was silenced in the cells by transfection of short interfering RNA and cell proliferation and apoptosis were evaluated by a tetrazolium salt-based assay and flow cytometry, respectively. CIP2A knockdown inhibited proliferation and induced apoptosis in RPMI-8226 and NCI-H929 cells and decreased the phosphorylation of phosphoinositide 3-kinase (PI3K) p85, AKT1, and mammalian target of rapamycin (mTOR) without affecting total protein levels. Treatment of CIP2A-depletion cells with insulin-like growth factor 1 decreased the effects of CIP2A inhibition on cell viability and apoptosis. These results indicate that CIP2A modulates myeloma cell proliferation and apoptosis via PI3K/AKT/mTOR signaling and suggest that it can potentially serve as a drug target for the treatment of multiple myeloma.
\end{abstract}

\section{Introduction}

Multiple myeloma-also known as plasma cell myeloma, myelomatosis, or Kahler's disease-is a cancer of plasma cells, a type of white blood cell that produces antibodies [1]. According to the National Cancer Institute Surveillance, Epidemiology, and End Results Program (http://seer.cancer .gov/), the number of new multiple myeloma cases is 6.3 per 100,000 men and women per year; mortality rate is 3.3 deaths per 100,000 men and women per year; and the 5-year survival rate is $46.6 \%$. Multiple myeloma is the second most prevalent blood cancer (10\%) after non-Hodgkin's lymphoma [2] and represents approximately $1 \%$ of all cancers and $2 \%$ of all deaths from cancer. It is therefore necessary to identify critical targets in multiple myeloma neoplasia and progression so that effective treatments can be developed.

The phosphoinositide 3-kinase (PI3K) signaling pathway mediates multiple myeloma cell proliferation and apoptosis and its activation increases with progression of the disease [3]. AKT is a downstream effector of PI3K that can be deactivated by the protein phosphatase $2 \mathrm{~A}$ (PP2A) complex via dephosphorylation of Thr308 and Ser473 [4]. Activated AKT modulates the phosphorylation status of various substrates involved in cell survival, cell cycle progression, and cellular growth, including mammalian target of rapamycin (mTOR) [5]. PI3K/AKT/mTOR signaling regulates a broad range of cellular processes including survival, proliferation, growth, metabolism, angiogenesis, and metastasis and is hyperactivated or dysregulated in many cancer types [6]. The regulation of the PI3K/AKT/mTOR pathway is not fully understood with respect to multiple myeloma; however, in most cell types, it is controlled by phosphatases such as PP2A $[7,8]$. For example, PP2A inhibition activates PI3K/AKT signaling through regulating the phosphorylation of AKT at Ser473 in SV40ST-induced human cancer cell transformation [8].

Cancerous inhibitor of PP2A (CIP2A) - also known as KIAA1524 or p90 tumor-associated antigen-is a human oncoprotein that is overexpressed in human neck and head carcinomas as well as breast, colon, and gastric cancers [5, 
9-12]. Inhibiting CIP2A decreases cancer cell viability and anchorage-independent growth and induces apoptosis $[5,9]$. In human malignancies, CIP2A stabilizes c-myc by inhibiting PP2A-mediated myc dephosphorylation at Ser62 [9]. In addition to blocking c-myc degradation, CIP2A expression is regulated by a positive feedback loop involving c-myc [10].

The precise function of CIP2A in human multiple myeloma has never been reported. The present study investigated the role of CIP2A in cell proliferation and apoptosis in the human multiple myeloma cell lines RPMI-8226 and NCI-H929 and its potential regulation of PI3K/AKT/mTOR signaling. The results demonstrate that CIP2A inhibition suppresses proliferation and induces apoptosis in RPMI-8226 and NCI-H929 cells while inactivating the PI3K/AKT/mTOR pathway. These findings provide novel insight into the roles of CIP2A in multiple myeloma progression and suggest that CIP2A can serve as a target for therapeutic agents in the treatment of this disease.

\section{Materials and Methods}

2.1. Cell Culture. The human multiple myeloma cell lines RPMI-8226 and NCI-H929 were purchased from the American Type Culture Collection (Manassas, VA, USA) and grown in RPMI-1640 medium supplemented with $10 \%$ (v/v) fetal bovine serum (FBS). The culture was maintained at $37^{\circ} \mathrm{C}$ in a $5 \% \mathrm{CO}_{2}$ atmosphere.

2.2. Transfection and Insulin-Like Growth Factor 1 (IGF1) Treatment. The short interfering RNA (siRNA) used to inhibit CIP2A expression was obtained from Jima Biotech (Suzhou, China). The following double-stranded oligonucleotides were used: CIP2A, $5^{\prime}$-CUG UGG UUG UGU UUG CAC UTT- $3^{\prime}$, and scrambled, $5^{\prime}$-UUC UCC GAA CGU GUC ACG UTT-3 ${ }^{\prime}$. For transfection, $2 * 10^{5}$ cells were seeded in culture plates and transfected with CIP2A siRNA (siCIP2A) or scrambled siRNA (si-Scr) using Lipofectamine 2000 (Invitrogen, Carlsbad, CA, USA) according to the manufacturer's instructions. After $48 \mathrm{~h}$, cells were collected for analysis. IGF-1 (Sigma-Aldrich, Darmstadt, Germany) was used at a concentration of $100 \mathrm{ng} / \mathrm{mL}$.

\subsection{RNA Extraction and Quantitative Real-Time PCR ( $q R T$ -} $P C R$ ). Total RNA was extracted from cell samples using TRIzol reagent according to the manufacturer's protocol. RNA was reverse transcribed into cDNA using PrimeScript RT reagent kit with gDNA Eraser (Takara Bio, Dalian, China) in a $20 \mu \mathrm{L}$ reaction according to the manufacturer's protocol. Equal amounts of cDNA were used as template for qRT-PCR to detect the level of CIP2A expression relative to that of actin (endogenous control), which was quantitated on an Mx3000P Real-Time PCR System using the SYBR Premix Ex Taq II PCR kit (Takara Bio) and the following primers: actin-F, $5^{\prime}$ ACT TCA CAT CAC AGC TCC CC- $3^{\prime}$, and actin-R, $5^{\prime}$-GAA TAT AAT CCC AAG CGG TTT G-3', and CIP2A-F, $5^{\prime}-\mathrm{CTG}$ GTG AGA TAA TCA GCA ATT T- $3^{\prime}$, and CIP2A-R, $5^{\prime}$-CGA AAC ATT CAT CAG ACT TTT CA- $3^{\prime}$. Experiments were performed in duplicate and repeated twice. Fold induction of gene expression was calculated using the $2^{-\Delta \Delta \mathrm{Ct}}$ method.
2.4. Cell Viability Assay. Cell viability was assessed with the [3-(4,5-dimethylthiazol-2-yl)-5-(3-carboxymethoxyphenyl)2-(4-sulfophenyl)-2H-tetrazolium (MTS) assay $24 \mathrm{~h}$ after transfection of si-CIP2A of si-Scr. Cells were seeded in 96-well plates in RPMI-1640 medium supplemented with $10 \%$ FBS at a density of $2 \times 10^{3}$ cells/well and cultured for 48, 72, and $96 \mathrm{~h}$. Viability was measured using the CellTiter 96 AQueous One Solution Cell Proliferation Assay kit (Promega, Madison, WI, USA) according to the manufacturer's instructions. Briefly, $20 \mu \mathrm{L}$ CellTiter 96 AQueous One Solution reagent was added to each well, followed by incubation for $3 \mathrm{~h}$ at $37^{\circ} \mathrm{C}$. The absorbance at $490 \mathrm{~nm}$ was measured using a Model 680 microplate reader (Bio-Rad, Hercules, CA, USA). Each experiment was carried out with six replicates and was repeated three times.

2.5. Apoptosis Assay. Apoptosis was determined using an apoptosis detection kit (Multisciences, Hangzhou, China) according to the manufacturer's instructions. Briefly, cells were digested with trypsin and collected by centrifugation at $2000 \mathrm{rpm}$ for $5 \mathrm{~min}$. Pellets were washed twice with phosphate-buffered saline (PBS) and centrifuged at $2000 \mathrm{rpm}$ for $5 \mathrm{~min}$; $1-5 \times 10^{5}$ cells were collected and resuspended in $500 \mu \mathrm{L}$ binding buffer. A $5 \mu \mathrm{L}$ volume of annexin $\mathrm{V}$ fluorescein isothiocyanate and $5 \mu \mathrm{L}$ propidium iodide were added to the suspension at room temperature and away from light for $15 \mathrm{~min}$. Cells were sorted within $1 \mathrm{~h}$ by flow cytometry using an Accuri C6 instrument (BD Biosciences, Franklin Lakes, NJ, USA).

2.6. Western Blot Analysis. Total protein lysates were extracted from cells and equal amounts of total protein were resolved by $10 \%$ sodium dodecyl sulfate polyacrylamide gel electrophoresis and transferred to a nitrocellulose membrane. Nonspecific binding was blocked with $5 \%$ milk in tris-buffered saline with Tween 20, and membranes were incubated overnight at $4^{\circ} \mathrm{C}$ with primary antibodies against the following proteins: CIP2A (1:1000), AKT1 (1:2000), phosphorylated AKT1 (p-AKT1) (Ser473) (1:1500), PI3K p85 (1:500), p-PI3K p85 (Tyr458) (1:1000), mTOR (1:1000), p-mTOR (Ser2448) (1:2000), and poly(ADP-ribose) polymerase (PARP, 1:1500) (all from Cell Signaling Technology, Danvers, MA, USA). Membranes were then incubated with horseradish peroxidase-conjugated secondary antibody and protein bands were detected by enhanced chemiluminescence (Thermo Scientific, Waltham, MA, USA).

2.7. Statistical Analysis. Data are expressed as the mean \pm SD and were analyzed using SPSS v. 19.0 software (SPSS Inc., Chicago, IL, USA). Differences between groups were assessed using Student's $t$-test. $P<0.05$ was considered statistically significant.

\section{Results}

3.1. siRNA Knockdown of CIP2A Expression. RPMI-8226 and NCI-H929 cells were transiently transfected with si-CIP2A or si-Scr at different concentrations $(25,50$, and $100 \mathrm{nM})$ and analyzed by qRT-PCR $24 \mathrm{~h}$ later. CIP2A mRNA expression 


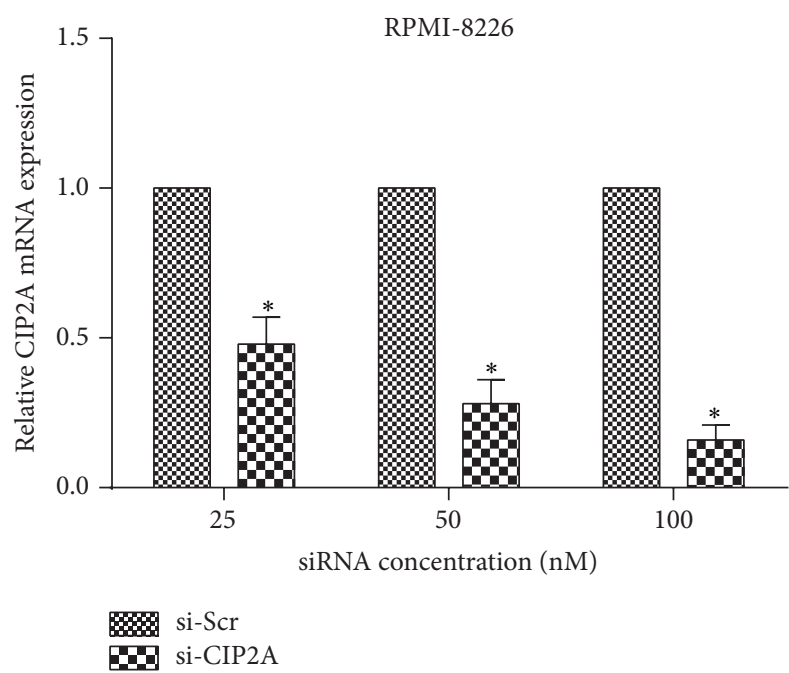

(a)

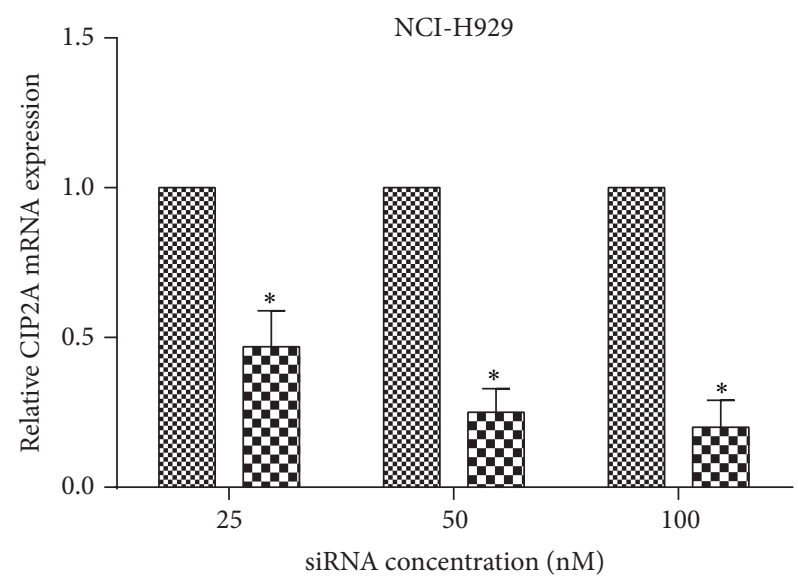

8i-Scr Di-CIP2A

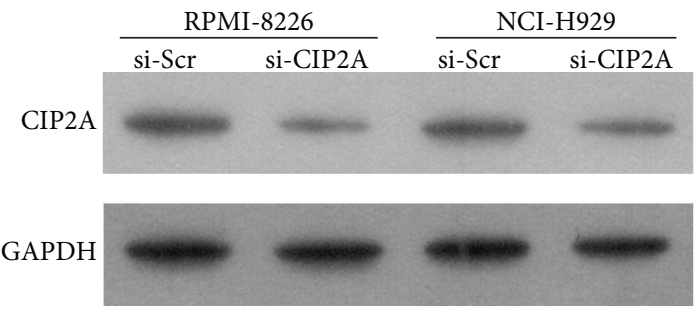

(c)

FIGURE 1: CIP2A expression following CIP2A knockdown. ((a) and (b)) CIP2A mRNA expression level in RPMI-8226 (a) and NCI-H929 (b) cells after transfection of indicated concentrations of si-CIP2A or si-Scr, as detected by qRT-PCR. Data are expressed as mean \pm SD. ${ }^{*} P<0.05$. (c) CIP2A protein expression after $100 \mathrm{nM}$ si-CIP2A or si-Sci transfection, as determined by western blotting. Glyceraldehyde-3-phosphate dehydrogenase (GAPDH) served as a loading control.

level in RPMI- 8226 cells decreased by $52 \%, 72 \%$, and $84 \%$ after si-CIP2A transfection at 25, 50, and $100 \mathrm{nM}$, respectively (Figure 1(a)). CIP2A mRNA expression level in NCIH929 cells decreased by $53 \%, 75 \%$, and $80 \%$ after si-CIP2A transfection at 25, 50, and $100 \mathrm{nM}$, respectively (Figure 1(b)). CIP2A protein expression was examined in cells $48 \mathrm{~h}$ after siRNA transfection. The protein level was almost completely suppressed by $100 \mu \mathrm{M}$ si-CIP2A treatment in both RPMI8226 and NCI-H929 cells (Figure 1(c)); this concentration was therefore used in subsequent experiments.

3.2. CIP2A Silencing Inhibits Multiple Myeloma Cell Proliferation. The role of CIP2A in human multiple myeloma was investigated by knocking down CIP2A expression in RPMI8226 and NCI-H929 cells and evaluating the effect on cell proliferation. Loss of CIP2A expression in RPMI-8226 cells inhibited cell viability by $24 \%, 32 \%$, and $38 \%$ at 48,72 , and $96 \mathrm{~h}$, respectively, after transfection (Figure 2(a)). Loss of CIP2A expression in NCI-H929 cells inhibited cell viability by $22 \%, 35 \%$, and $50 \%$ at 48,72 , and $96 \mathrm{~h}$, respectively, after transfection (Figure 2(b)).

3.3. CIP2A Inhibition Induces Multiple Myeloma Cell Apoptosis. The effect of CIP2A inhibition on apoptosis was examined by flow cytometry. The apoptotic fraction was higher upon transfection of si-CIP2A as compared to si-Scr both in RPMI-8226 and in NCI-H929 cells (Figure 3(a)); specifically, the percentage of early apoptotic cells was higher in cells depletion in CIP2A than in control cells both in RPMI-8226 and in NCI-H929 cells (Figure 3(b)). To further evidence whether si-CIP2A induced apoptosis, we detected the cleavage of PARP. As shown in Figure 3(c), the cleavage of PARP was more obvious in cells depletion in CIP2A than in control cells both in RPMI-8226 and in NCI-H929 cells.

3.4. CIP2A Knockdown Inactivates PI3K/AKT/mTOR Signaling. To investigate the mechanism underlying the effect of CIP2A on RPMI-8226 and NCI-H929 cell proliferation and apoptosis, we evaluated the expression of key proteins in the $\mathrm{PI} 3 \mathrm{~K} / \mathrm{AKT} / \mathrm{mTOR}$ signaling pathway $72 \mathrm{~h}$ after si-CIP2A or si-Scr transfection by western blotting. CIP2A knockdown decreased the phosphorylation level of PI3K p85, AKT1, and mTOR without altering total protein levels both in RPMI-8226 and in NCI-H929 cells (Figure 4), suggesting that pathway activation but not the expression of individual components was affected by loss of CIP2A.

3.5. IGF-1 Treatment Decreases the Effects of CIP2A Knockdown in Multiple Myeloma Cells. To clarify the role of $\mathrm{PI} 3 \mathrm{~K} / \mathrm{AKT} / \mathrm{mTOR}$ signaling in the regulation of multiple 


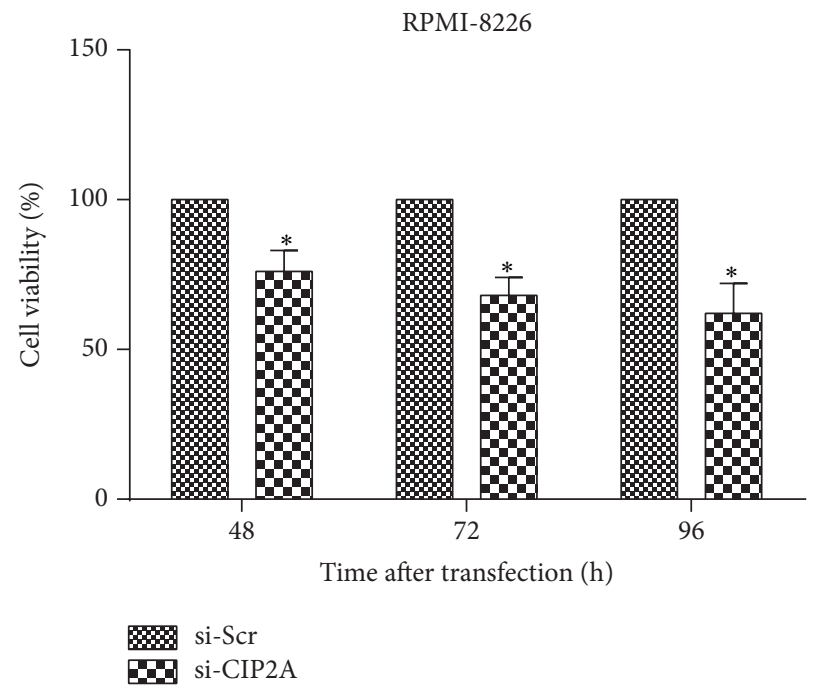

(a)

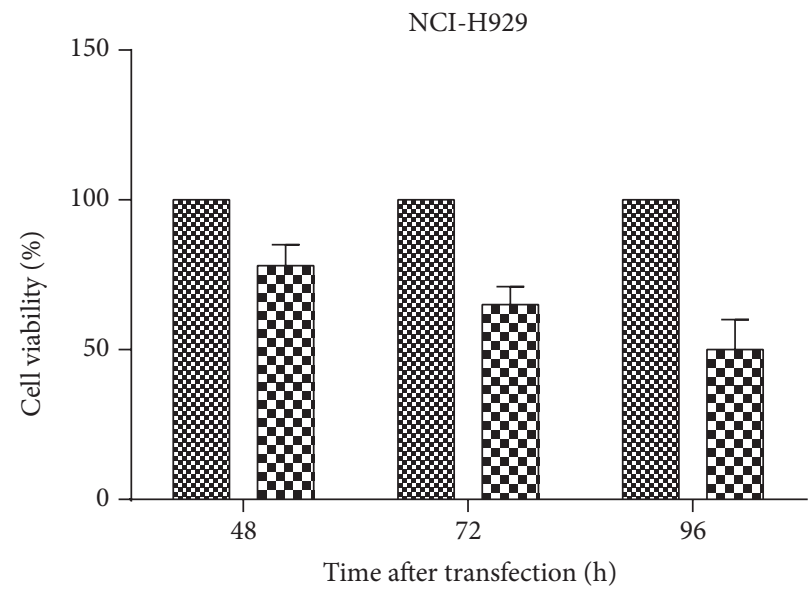

\%ి si-Scr Di-CIP2A

(b)

FIGURE 2: Effect of CIP2A knockdown on RPMI-8226 (a) and NCI-H929 (b) cell proliferation. Viable cells were counted 48, 72, and 96 h after transfection. Data are expressed as mean \pm SD. ${ }^{*} P<0.05$.

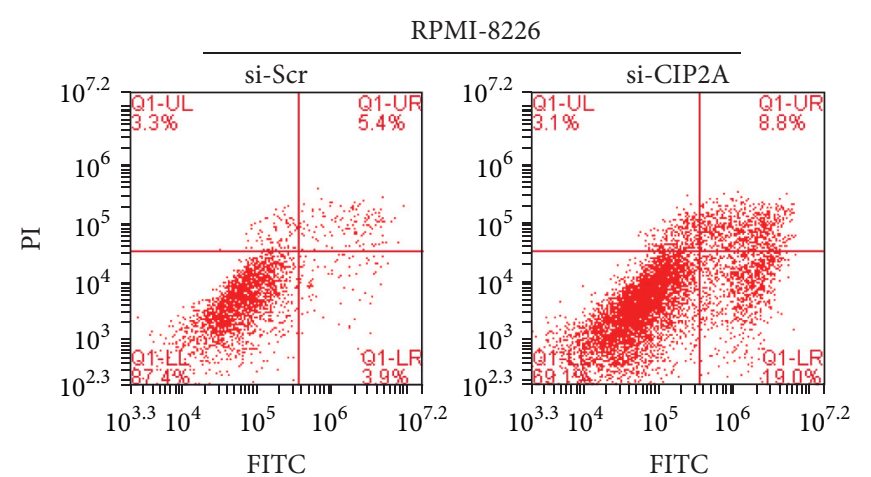

(a)

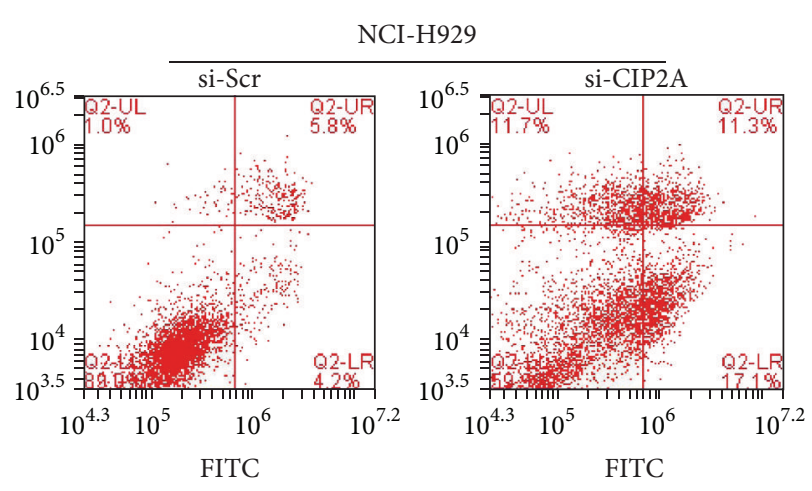

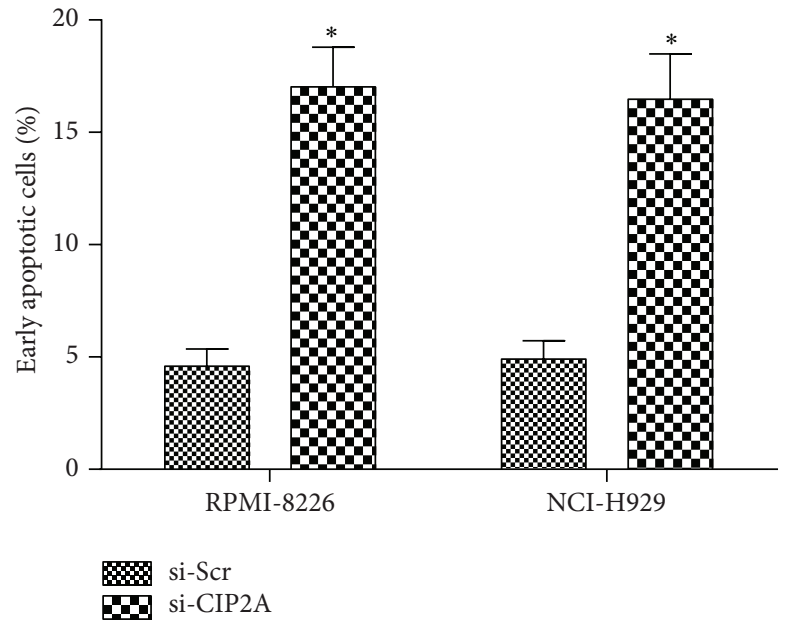

(b)

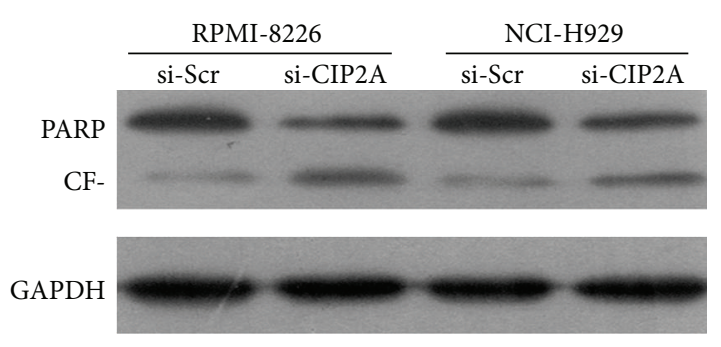

(c)

Figure 3: Effect of CIP2A knockdown on multiple myeloma cell apoptosis. (a) Representative image of a flow cytometry scatterplot of apoptotic RPMI-8226 and NCI-H929 cells labeled with fluorescein isothiocyanate (FITC) and propidium iodide [13] and sorted 72 h after transfection with si-CIP2A or si-Scr. (b) Quantitative analysis of early apoptotic cells after transfection with si-CIP2A or si-Scr. Data are expressed as mean \pm SD. ${ }^{*} P<0.05$. (c) The cleavage of PARP detected by western blot. CF: cleaved fragment. 


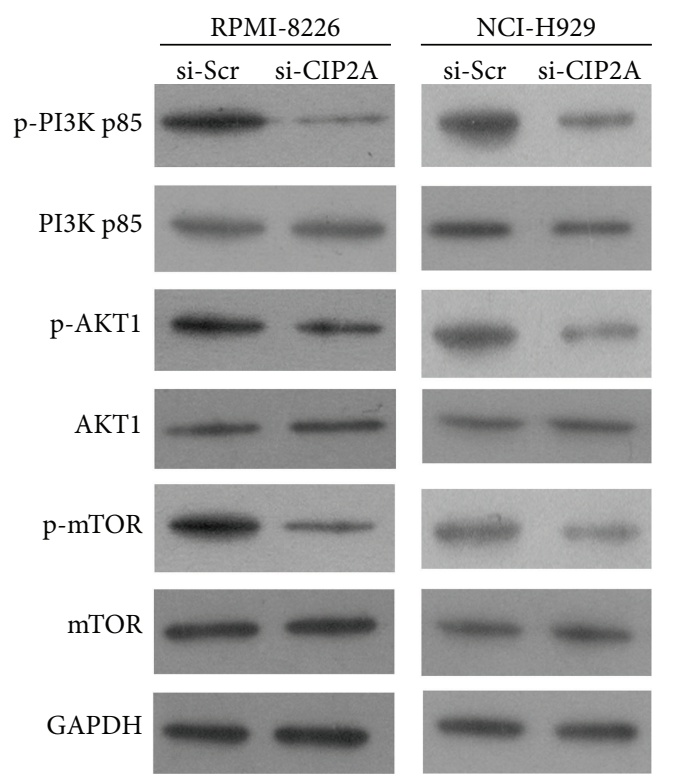

FIgURE 4: Effect of CIP2A knockdown on the expression level of PI3K/AKT/mTOR signaling components. The protein expression levels of nonphosphorylated and phosphorylated PI3K p85, AKT1, and mTOR in RPMI-8226 and NCI-H929 cells transfected with si-CIP2A or si-Scr were evaluated by western blotting, with glyceraldehyde-3-phosphate dehydrogenase (GAPDH) used as a loading control.

myeloma cell proliferation and apoptosis by CIP2A, we evaluated the effect of IGF-1 which could activate PI3K [14] on RPMI-8226 and NCI-H929 cells. Firstly, we tested the expression level of $\mathrm{PI} 3 \mathrm{~K} / \mathrm{AKT} / \mathrm{mTOR}$ signaling components upon IGF-1 treatment in RPMI-8226 and NCI-H929 cells that are knocked down by si-CIP2A. As shown in Figure 5, the decreased phosphorylation of PI3K p85, AKT1, and mTOR caused by si-CIP2A was increased by IGF-1 treatment. Then we evaluated the effect of IGF-1 on cell proliferation and apoptosis in RPMI-8226 and NCI-H929 cells that are knocked down by si-CIP2A. The results showed that treatment with IGF-1 $(100 \mathrm{ng} / \mathrm{mL})$ reversed the decrease in cell viability caused by CIP $2 A$ knockdown (Figures 6(a) and $6(\mathrm{~b})$ ) and reduced the rate of apoptosis relative to untreated si-CIP2A-transfected cells (Figures 6(c) and 6(d)). In addition, treatment with IGF-1 reversed the increase in the cleavage of PARP caused by CIP2A knockdown (Figure 6(e)). Taken together, the results indicate that CIP2A knockdown modulates multiple myeloma cell proliferation and apoptosis via inhibition of PI3K/AKT/mTOR signaling.

\section{Discussion}

CIP2A is a human oncoprotein that was initially identified in patients with gastric and liver cancers, which has unknown function due to the lack of the homology to any known proteins [12]. CIP2A is overexpressed in several types of cancer cell $[5,10,15-18]$, and its utility as a prognostic marker has been established in various solid and hematological cancers, including esophageal squamous cell and non-small

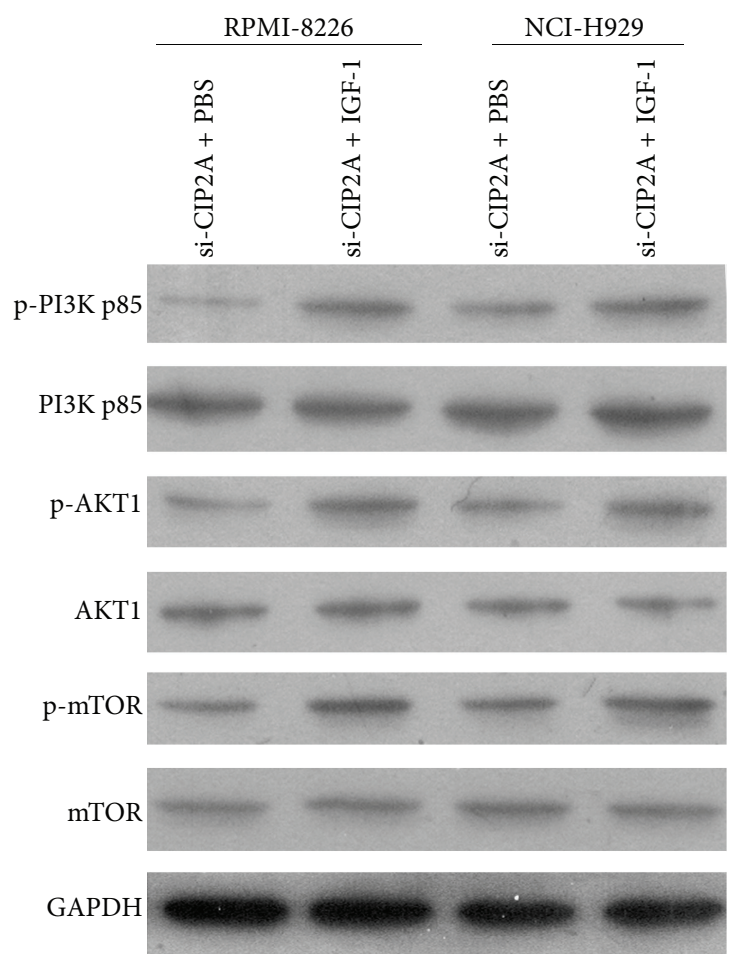

FIgURE 5: Effect of IGF-1 treatment on the expression level of $\mathrm{PI} 3 \mathrm{~K} / \mathrm{AKT} / \mathrm{mTOR}$ signaling components in RPMI-8226 and NCIH929 cells that are knocked down by si-CIP2A. The protein expression levels of nonphosphorylated and phosphorylated PI3K p85, AKT1, and mTOR in RPMI-8226 and NCI-H929 cells that are knocked down by si-CIP2A treated with PBS or IGF-1 were evaluated by western blotting, with glyceraldehyde-3-phosphate dehydrogenase (GAPDH) used as a loading control.

cell lung carcinoma; breast, gastric, bladder, ovarian, tongue, hepatocellular, and colon cancers; and chronic myelogenous leukemia $[5,10,15-21]$. The present study investigated the role of CIP2A in human multiple myeloma using the RPMI-8226 and NCI-H929 cell lines. We found that CIP2A knockdown inhibited proliferation and induced apoptosis, suggesting that CIP2A acts as an oncoprotein in RPMI-8226 and NCI-H929 cells. This is in agreement with results of previous studies; for instance, in gastric cancer, CIP2A was detected in tumor tissues but not in normal gastric mucosa, and depleting CIP2A expression suppressed the growth of tumor cell lines [11]. In addition, CIP2A is overexpressed in esophageal squamous cell carcinoma relative to normal tissues, and CIP2A knockdown was found to inhibit tumor cell growth [19]. However, our results on the effect of CIP2A on cell apoptosis are inconsistent with results of previous studies; for instance, in cervical cancer, breast cancer, and hepatocellular carcinoma, CIP2A knockdown does not induce significant apoptosis $[9,22,23]$. It is difficult to explain the discrepancy based on the present study. However, all these results showed that CIP2A function is not conserved across different cancer cell types. 


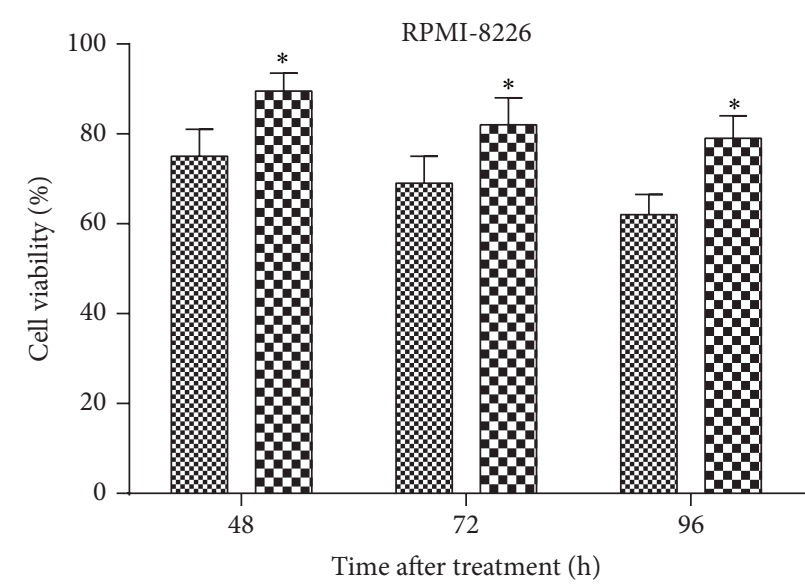

38 si-CIP2A + PBS

Di-CIP2A + IGF-1

(a)

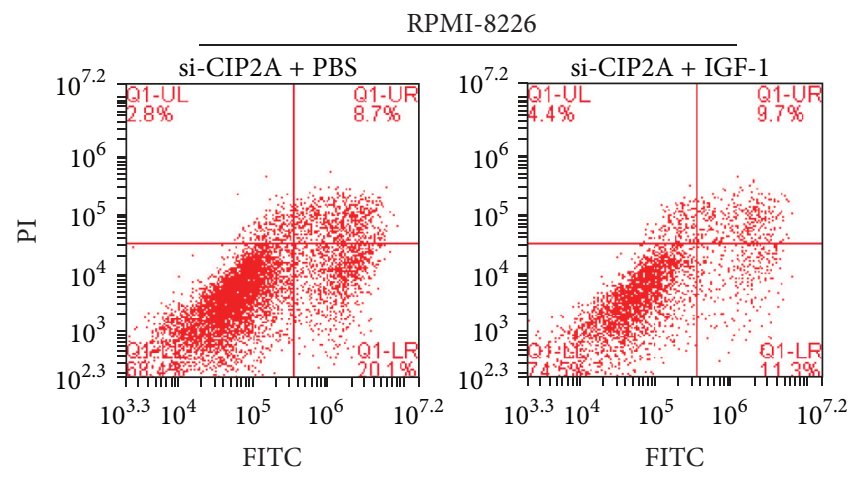

(c)

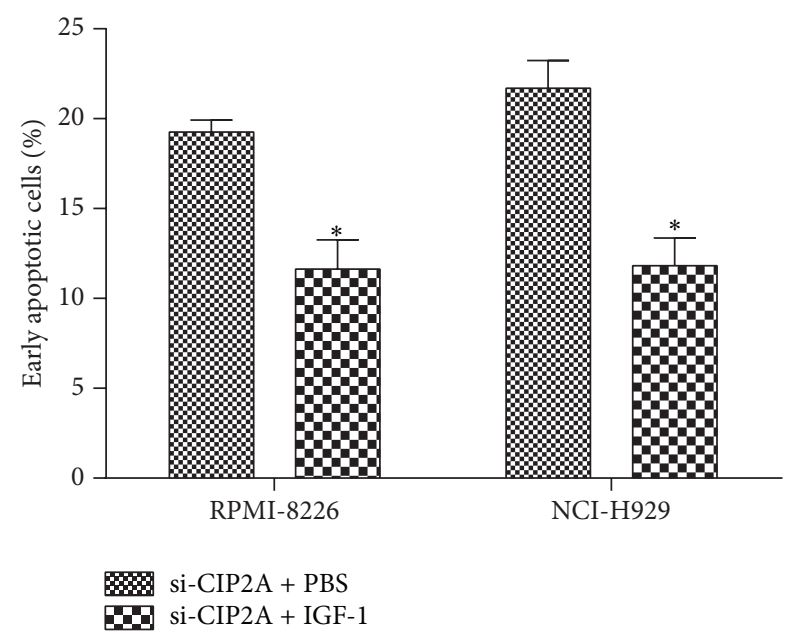

(d)

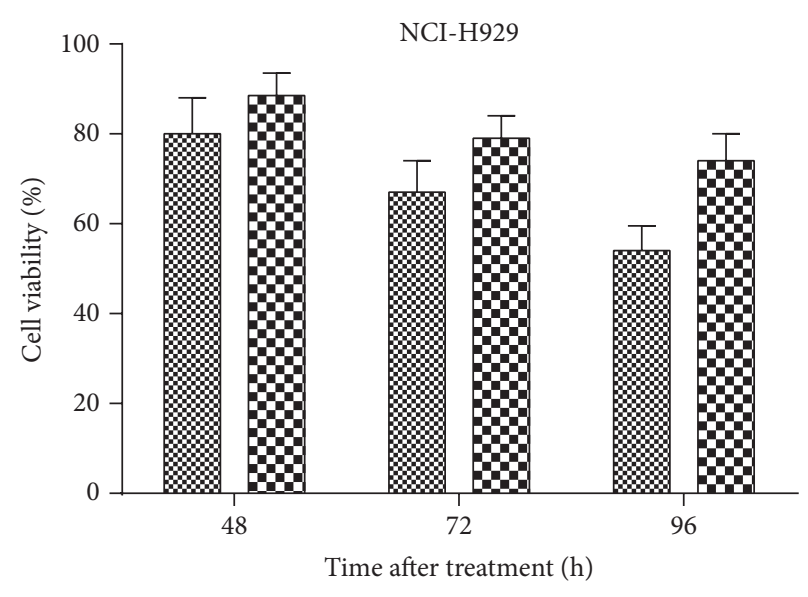

\%

Di-CIP2A + IGF-1

(b)
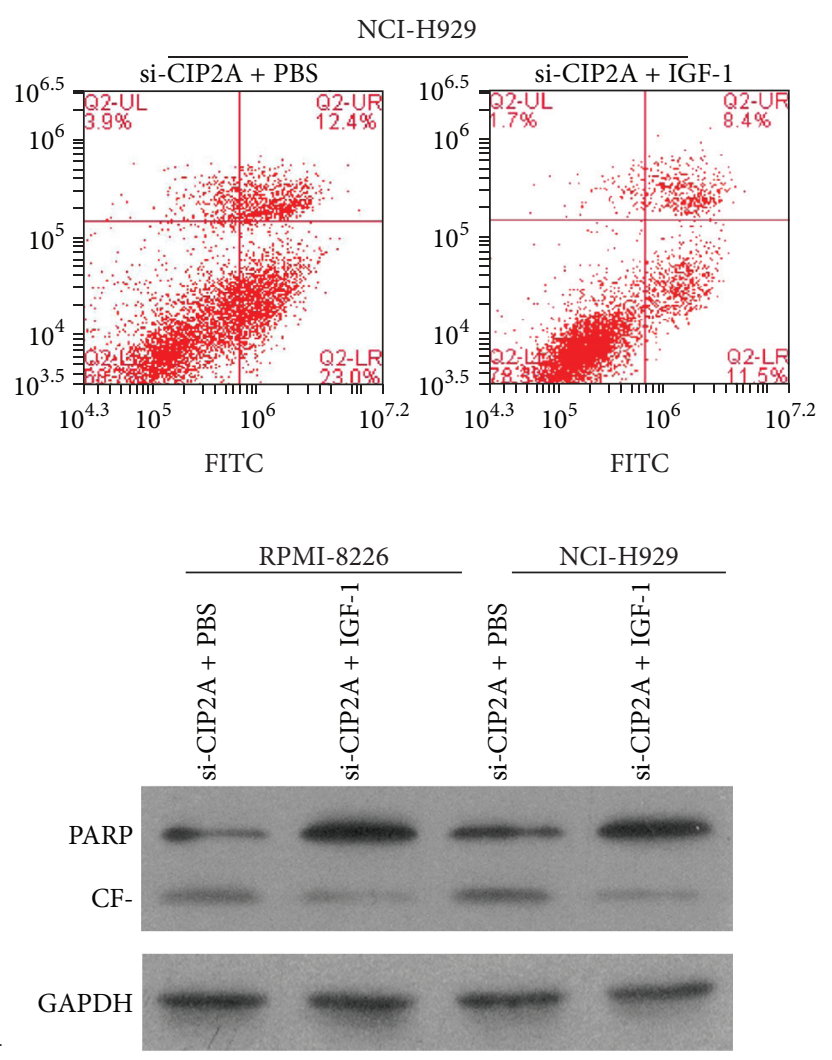

(e)

FIGURE 6: IGF-1 treatment reverses the effects of CIP2A knockdown in RPMI-8226 and NCI-H929 cells. ((a) and (b)) RPMI-8226 (a) and NCI-H929 (b) cells transfected with si-CIP2A were treated with PBS (control) or the PI3K activator IGF-1, and the effect on cell viability was determined by the MTS assay. The results were normalized to si-Scr transfected cells. ((c) and (d)) Effect of IGF-1 treatment on RPMI-8226 and NCI-H929 cell apoptosis. Flow cytometry scatterplot of apoptotic RPMI-8226 and NCI-H929 cells labeled with fluorescein isothiocyanate (FITC) and propidium iodide and sorted $72 \mathrm{~h}$ after transfection with si-CIP2A followed by treatment with PBS or IGF-1 (c) and quantitative analysis of early apoptotic cells after transfection with si-CIP2A followed by treatment with PBS or IGF-1 (d). (e) The cleavage of PARP in each group detected by western blot. CF: cleaved fragment. Data are expressed as mean \pm SD of three independent experiments. ${ }^{*} P<0.05$. 


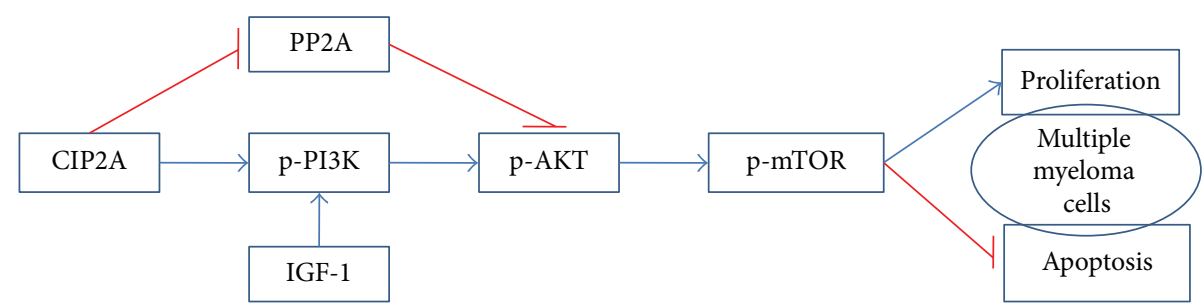

FIgURE 7: The scheme illustrating the mechanism by which knockdown CIP2A induces apoptosis and antiproliferation including the counteraction of IGF-1 and PP2A in myeloma cells. Blue arrows indicate activation. Red T-bars indicate suppression.

Based on the experimental evidence that CIP2A has clinical relevance in the progression of the disease it has been regarded that CIP2A inhibitors have potential for use in the treatment in cancers [24]. So far CIP2A has been targeted in a limited number of cancers, such as hepatocellular carcinoma [25], as well as oral cancer [26]. The expression of CIP2A also could be inhibited by natural compounds and fusogenicoligoarginine peptide-mediated delivery of siRNAs for gene silencing and erlotinib derivatives [24]. And high expression of CIP2A has also been proposed as a useful biomarker that predicts therapeutic response to chemotherapeutics such as bortezomib, erlotinib, afatinib, checkpoint kinase 1 inhibitors, and prosenescence based therapies [13, 27-29]. Based on these previous results, we predicted that CIP2A might serve as a potential target for therapeutic agents developed for the treatment of multiple myeloma.

$\mathrm{PI} 3 \mathrm{~K} / \mathrm{AKT} / \mathrm{mTOR}$ signaling plays a critical role in the malignant transformation of human tumors and their subsequent growth, proliferation, and metastasis [30]. In the present study, we found that CIP2A knockdown led to decreased phosphorylation of PI3K p85 at Tyr458, AKT1 at Ser473, and mTOR at Ser2448, and treatment with the IGF-1 which could activate PI3K [14] decreased the effect of CIP2A knockdown on the expression level of PI3K/AKT/mTOR signaling components. CIP2A overexpression can induce an increase in AKT phosphorylation in head and neck squamous cell carcinoma [22] and in liver cancer cells on both Thr308 and Ser473 of AKT; moreover, CIP2A inhibition induced the dephosphorylation of Ser473 in liver cancer and triple-negative breast cancer and lung cancer cells [23, 31, 32]. In addition, the inhibition of PP $2 \mathrm{~A}$ which could be inhibited by CIP2A activates PI3K/AKT signaling through regulating the phosphorylation of AKT at Ser473 in SV40STinduced human cancer cell transformation [8]. Based on these results, we predicted that Ser473 of AKT is a direct substrate of PP2A in human multiple myeloma using the RPMI-8226 and NCI-H929 cell lines. As an inhibitor of $\mathrm{PP} 2 \mathrm{~A}$ which is a serine/threonine phosphatase, there is no evidence showing CIP2A can regulate the phosphorylation of PI3K p85 at Tyr458 or PP2A can directly dephosphorylate tyrosine phosphorylation sites of PI3K p85. Therefore, $\mathrm{p}$ PI3K p85 cannot be a direct substrate of PP2A, although phosphorylation of PI3K p85 at Tyr45 could be decreased by CIP2A knockdown. Previous studies indicated the phosphorylation of mTOR at Ser2448 was also not a direct substrate of PP2A $[33,34]$. These results indicated that CIP2A could regulate $\mathrm{PI} 3 \mathrm{~K} / \mathrm{AKT} / \mathrm{mTOR}$ signaling pathway, but the regulatory mechanisms between CIP2A and PI3K/AKT/ mTOR signaling pathway seemed to be complex.

In conclusion, the results of our study indicate that CIP2A acts as an oncoprotein in human multiple myeloma. Knocking down CIP2A expression inhibited cell proliferation and induced early apoptosis via inactivation of PI3K/AKT/mTOR signaling, as shown in Figure 7. Therefore, CIP2A can serve as a potential target for therapeutic agents developed for the treatment of multiple myeloma.

\section{Competing Interests}

The authors have no competing interests pertaining to this paper.

\section{Authors' Contributions}

Xi Yang and Yaping Zhang contributed equally to this study.

\section{References}

[1] M. S. Raab, K. Podar, I. Breitkreutz, P. G. Richardson, and K. C. Anderson, "Multiple myeloma," The Lancet, vol. 374, no. 9686, pp. 324-339, 2009.

[2] C. D. Collins, "Problems monitoring response in multiple myeloma," Cancer Imaging, vol. 5, pp. S119-S126, 2005.

[3] J.-H. Hsu, Y. Shi, S. Krajewski et al., "The AKT kinase is activated in multiple myeloma tumor cells," Blood, vol. 98, no. 9, pp. 28532855, 2001.

[4] S. Andrabi, O. V. Gjoerup, J. A. Kean, T. M. Roberts, and B. Schaffhausen, "Protein phosphatase $2 \mathrm{~A}$ regulates life and death decisions via Akt in a context-dependent manner," Proceedings of the National Academy of Sciences of the United States of America, vol. 104, no. 48, pp. 19011-19016, 2007.

[5] C. Côme, A. Laine, M. Chanrion et al., "CIP2A is associated with human breast cancer aggressivity," Clinical Cancer Research, vol. 15, no. 16, pp. 5092-5100, 2009.

[6] T. Ersahin, N. Tuncbag, and R. Cetin-Atalay, “The PI3K/AKT/ mTOR interactive pathway," Molecular Biosystems, vol. 11, no. 7, pp. 1946-1954, 2015.

[7] Q. Zhang and F. X. Claret, "Phosphatases: the new brakes for cancer development?" Enzyme Research, vol. 2012, Article ID 659649, 11 pages, 2012.

[8] A. A. Sablina, M. Hector, N. Colpaert, and W. C. Hahn, "Identification of PP2A complexes and pathways involved in cell transformation," Cancer Research, vol. 70, no. 24, pp. 10474-10484, 2010. 
[9] M. R. Junttila, P. Puustinen, M. Niemelä et al., "CIP2A inhibits PP2A in human malignancies," Cell, vol. 130, no. 1, pp. 51-62, 2007.

[10] A. Khanna, C. Böckelman, A. Hemmes et al., "MYC-dependent regulation and prognostic role of CIP2A in gastric cancer," Journal of the National Cancer Institute, vol. 101, no. 11, pp. 793-805, 2009.

[11] W. Li, Z. Ge, C. Liu et al., "CIP2A is overexpressed in gastric cancer and its depletion leads to impaired clonogenicity, senescence, or differentiation of tumor cells," Clinical Cancer Research, vol. 14, no. 12, pp. 3722-3728, 2008.

[12] L. S. Hoo, J. Y. Zhang, and E. K. L. Chan, "Cloning and characterization of a novel $90 \mathrm{kDa}$ 'companion' auto-antigen of $\mathrm{p} 62$ overexpressed in cancer," Oncogene, vol. 21, no. 32, pp. 50065015, 2002.

[13] A. Khanna and J. E. Pimanda, "Clinical significance of cancerous inhibitor of protein phosphatase 2A in human cancers," International Journal of Cancer, vol. 138, no. 3, pp. 525-532, 2016.

[14] G. Shen, R. Wu, B. Liu et al., "Upstream and downstream mechanisms for the promoting effects of IGF-1 on differentiation of spermatogonia to primary spermatocytes," Life Sciences, vol. 101, no. 1-2, pp. 49-55, 2014.

[15] C. Böckelman, H. Lassus, A. Hemmes et al., "Prognostic role of CIP2A expression in serous ovarian cancer," British Journal of Cancer, vol. 105, no. 7, pp. 989-995, 2011.

[16] Q.-Z. Dong, Y. Wang, X.-J. Dong et al., “CIP2A is overexpressed in non-small cell lung cancer and correlates with poor prognosis," Annals of Surgical Oncology, vol. 18, no. 3, pp. 857-865, 2011.

[17] Y. Xue, G. Wu, X. Wang et al., "CIP2A is a predictor of survival and a novel therapeutic target in bladder urothelial cell carcinoma," Medical Oncology, vol. 30, no. 1, article 406, 2013.

[18] H. He, G. Wu, W. Li, Y. Cao, and Y. Liu, "CIP2A is highly expressed in hepatocellular carcinoma and predicts poor prognosis," Diagnostic Molecular Pathology, vol. 21, no. 3, pp. 143-149, 2012.

[19] W. Qu, W. Li, L. Wei, L. Xing, X. Wang, and J. Yu, "CIP2A is overexpressed in esophageal squamous cell carcinoma," Medical Oncology, vol. 29, no. 1, pp. 113-118, 2012.

[20] C. M. Lucas, R. J. Harris, A. Giannoudis, M. Copland, J. R. Slupsky, and R. E. Clark, "Cancerous inhibitor of PP2A (CIP2A) at diagnosis of chronic myeloid leukemia is a critical determinant of disease progression," Blood, vol. 117, no. 24, pp. 6660-6668, 2011.

[21] A. Wiegering, C. Pfann, F. W. Uthe et al., "CIP2A influences survival in colon cancer and is critical for maintaining Myc expression," PLoS ONE, vol. 8, no. 10, Article ID e75292, 2013.

[22] Y.-C. Lin, K.-C. Chen, C.-C. Chen, A.-L. Cheng, and K.-F. Chen, "CIP2A-mediated Akt activation plays a role in bortezomibinduced apoptosis in head and neck squamous cell carcinoma cells," Oral Oncology, vol. 48, no. 7, pp. 585-593, 2012.

[23] L.-M. Tseng, C.-Y. Liu, K.-C. Chang, P.-Y. Chu, C.-W. Shiau, and K.-F. Chen, "CIP2A is a target of bortezomib in human triple negative breast cancer cells," Breast Cancer Research, vol. 14, no. 2, 2012.

[24] P. De, J. Carlson, B. Leyland-Jones, and N. Dey, "Oncogenic nexus of cancerous inhibitor of protein phosphatase $2 \mathrm{~A}$ (CIP2A): an oncoprotein with many hands," Oncotarget, vol. 5, no. 13, pp. 4581-4602, 2014.

[25] H.-C. Yu, H.-J. Chen, Y.-L. Chang et al., "Inhibition of CIP2A determines erlotinib-induced apoptosis in hepatocellular carcinoma," Biochemical Pharmacology, vol. 85, no. 3, pp. 356-366, 2013.
[26] L. Cantini, C. C. Attaway, B. Butler, L. M. Andino, M. L. Sokolosky, and A. Jakymiw, "Fusogenic-oligoarginine peptidemediated delivery of siRNAs targeting the CIP2A oncogene into oral cancer cells," PLoS ONE, vol. 8, no. 9, Article ID e73348, 2013.

[27] C.-Y. Liu, C.-W. Shiau, H.-Y. Kuo et al., "Cancerous inhibitor of protein phosphatase $2 \mathrm{~A}$ determines bortezomib-induced apoptosis in leukemia cells," Haematologica, vol. 98, no. 5, pp. 729738, 2013.

[28] T.-T. Chao, C.-Y. Wang, Y.-L. Chen et al., "Afatinib induces apoptosis in NSCLC without EGFR mutation through Elk-1mediated suppression of CIP2A," Oncotarget, vol. 6, no. 4, pp. 2164-2179, 2015.

[29] H.-C. Yu, M.-H. Hung, Y.-L. Chen et al., "Erlotinib derivative inhibits hepatocellular carcinoma by targeting CIP2A to reactivate protein phosphatase 2A," Cell Death and Disease, vol. 5, no. 7, Article ID e1359, 2014.

[30] S. Mabuchi, H. Kuroda, R. Takahashi, and T. Sasano, "The PI3K/ AKT/mTOR pathway as a therapeutic target in ovarian cancer," Gynecologic Oncology, vol. 137, no. 1, pp. 173-179, 2015.

[31] K.-F. Chen, C.-Y. Liu, Y.-C. Lin et al., "CIP2A mediates effects of bortezomib on phospho-Akt and apoptosis in hepatocellular carcinoma cells," Oncogene, vol. 29, no. 47, pp. 6257-6266, 2010.

[32] N. Lei, B. Peng, and J.-Y. Zhang, "CIP2A regulates cell proliferation via the AKT signaling pathway in human lung cancer," Oncology Reports, vol. 32, no. 4, pp. 1689-1694, 2014.

[33] S. Kumar and K. Tikoo, "Independent role of PP2A and mTORcl in palmitate induced podocyte death," Biochimie, vol. 112, pp. 73-84, 2015.

[34] D. Hartley and G. M. Cooper, "Role of mTOR in the degradation of IRS-1: regulation of PP2A activity," Journal of Cellular Biochemistry, vol. 85, no. 2, pp. 304-314, 2002. 


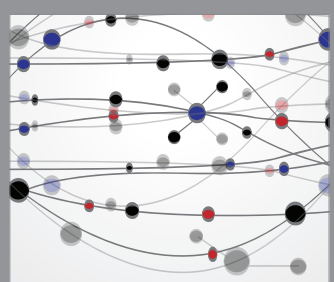

The Scientific World Journal
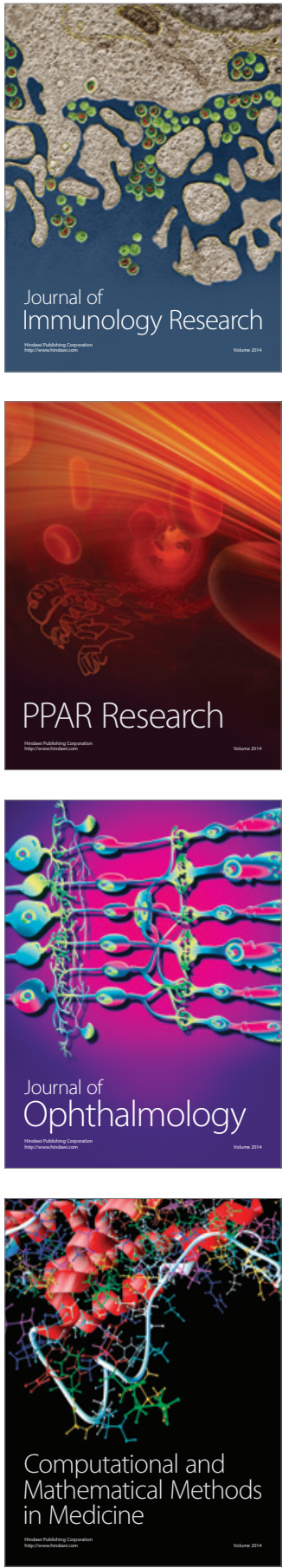

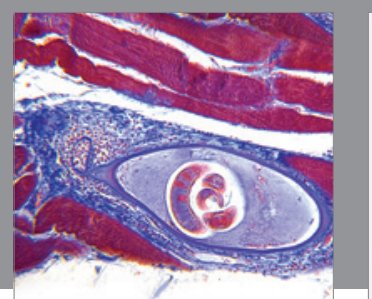

Gastroenterology Research and Practice

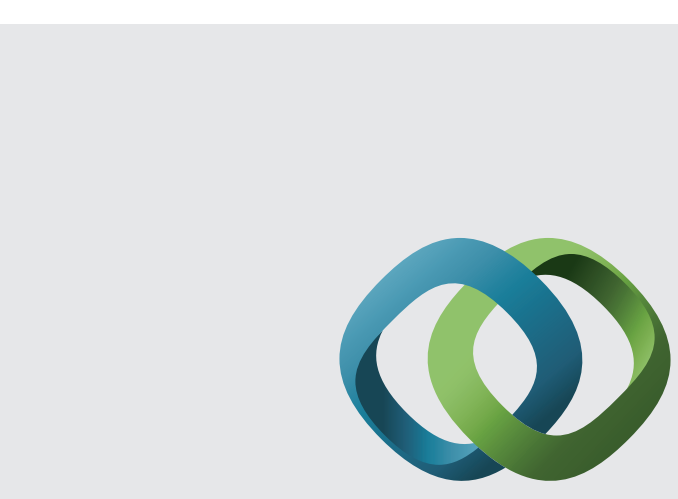

\section{Hindawi}

Submit your manuscripts at

http://www.hindawi.com
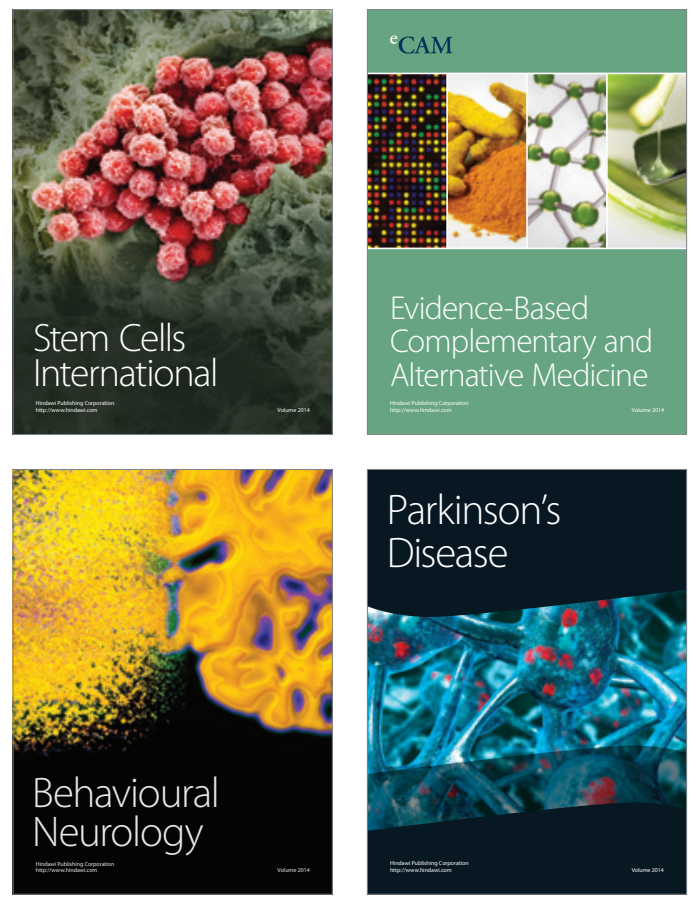
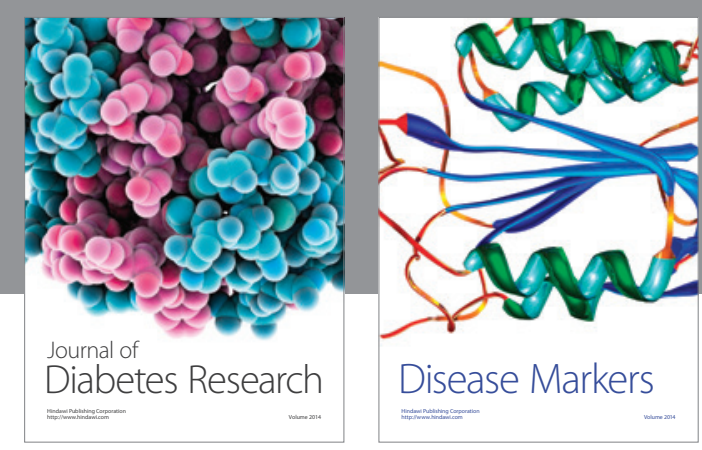

Disease Markers
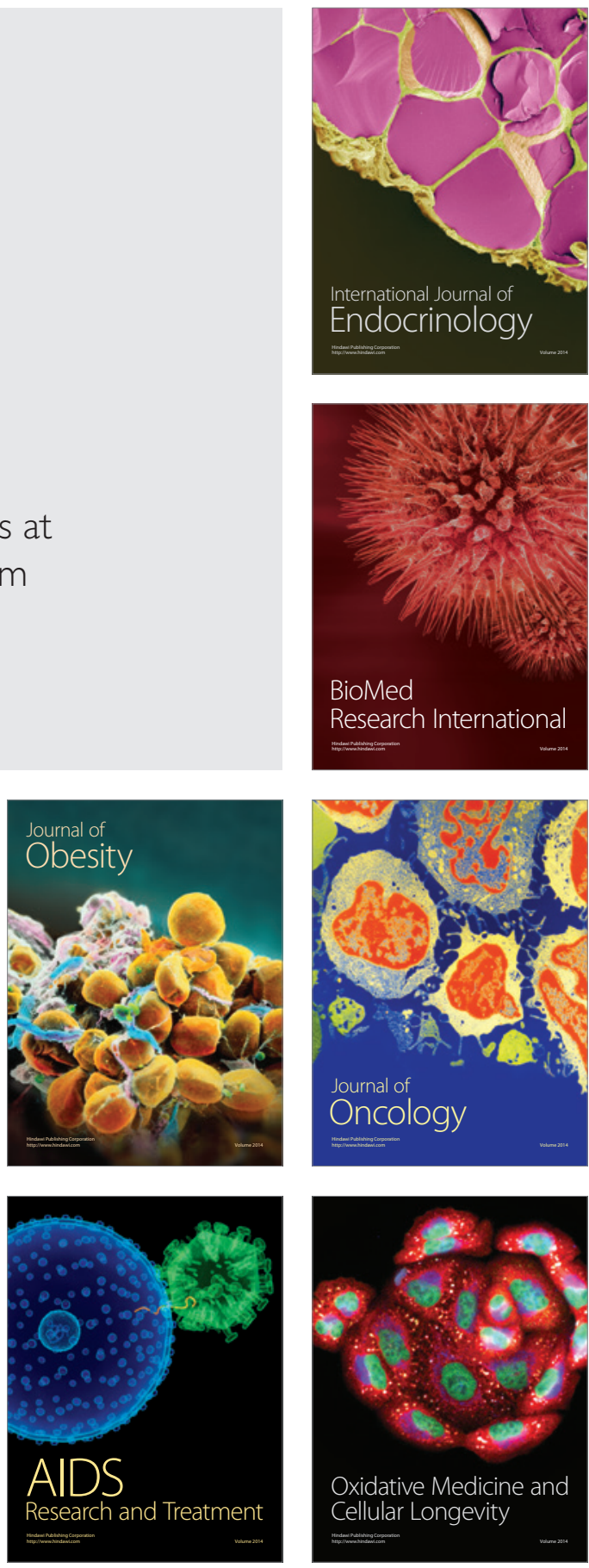